\title{
Probing the gluon Wigner distribution in diffractive dijet production
}

\author{
Yoshitaka Hatta* \\ Yukawa Institute for Theoretical Physics, Kyoto University, Sakyo-ku, Kyoto, 606-8502, Japan \\ E-mail: hatta@yukawa.kyoto-u.ac.jp
}

We study diffractive dijet production in lepton-nucleon scattering and in ultraperipheral protonnucleus collisions as a means to probe the phase space (Wigner) distribution of small-x gluons. We also discuss the small- $x$ behavior of the gluon orbital angular momentum distribution.

XXV International Workshop on Deep-Inelastic Scattering and Related Subjects 3-7 April 2017

University of Birmingham, $U K$

${ }^{*}$ Speaker. 


\section{Introduction}

In the study of nucleon tomography, it is often useful to consider the Wigner distribution $W(x, \boldsymbol{k}, \boldsymbol{b})[1]$ as the phase space distribution of partons inside the nucleon. In addition to the longitudinal momentum fraction $x$, the Wigner distribution depends on the transverse momentum $\boldsymbol{k}$ and the impact parameter $\boldsymbol{b}$ of partons. Such a five-dimensional distribution encodes the complete single-parton information of the nucleon, and can be considered as a 'mother distribution' as it reduces to the more familiar three-dimensional distributions, the transverse momentum dependent (TMD) distribution and the generalized parton distribution (GPD), upon integration over $\boldsymbol{b}$ and $k$, respectively. In addition to the Wigner distribution, two associated phase space distributions have been proposed. One is the generalized TMD (GTMD) distribution $G(x, \boldsymbol{k}, \boldsymbol{\Delta})$ [2] which is the Fourier transform of the Wigner distribution $\boldsymbol{b} \leftrightarrow \Delta$. The other is the Husimi distribution $H(x, \boldsymbol{k}, \boldsymbol{b})$ [3] obtained from the Wigner distribution via Gaussian smearing in both $\boldsymbol{b}$ and $\boldsymbol{k}$. These distributions provide a multifaceted insight into the complicated partonic structure of the nucleon.

Naturally, these five dimensional distributions contain richer physics than TMDs and GPDs combined. By separately studying the $\boldsymbol{k}$ and $\boldsymbol{b}$ distributions via TMD and GPD, one can not learn about the correlation between $\boldsymbol{b}$ and $\boldsymbol{k}$. Such a correlation is practically important, most prominently for the understanding the parton orbital angular momentum. Indeed, the proper, gauge-invariant definition of the canonical orbital angular momentum in QCD involves the Wigner or Husimi distribution for quarks and gluons $[4,5,3]$

$$
L_{q, g}=\int d x d \boldsymbol{b} d \boldsymbol{k}(\boldsymbol{b} \times \boldsymbol{k})_{z}\left\{\begin{array}{l}
W_{q, g}(x, \boldsymbol{k}, \boldsymbol{b}) \\
H_{q, g}(x, \boldsymbol{k}, \boldsymbol{b}) .
\end{array}\right.
$$

It is clear that three dimensional distributions which depend on either $\boldsymbol{b}$ or $\boldsymbol{k}$ do not give access to $L_{q, g}$.

Unfortunately, it is very difficult to measure these phase space distributions in QCD, or more generally, in any quantum theory. Because of this, previous studies on this subject have been mostly restricted to formal theoretical issues and simple model calculations, with little reference to experiments. There are however known examples in quantum optics [8] where one can experimentally measure the Wigner distribution. Here we show that the gluon phase space distribution at small- $x$ is actually measurable in lepton-nucleon and nucleon-nucleus scattering.

\section{Probing the phase space distributions in $e p$ and $p A$ collisions}

The gluon Wigner distribution is defined by

$$
x W(x, \boldsymbol{k}, \boldsymbol{b})=\frac{2}{P^{+}} \int \frac{d^{3} z}{(2 \pi)^{3}} \frac{d^{2} \boldsymbol{\Delta}}{(2 \pi)^{2}} e^{-i x P^{+} z^{-}-i \boldsymbol{k} \cdot \mathbf{z}}\left\langle P+\frac{\Delta}{2}\left|\operatorname{Tr}\left[U^{[+]} F^{+i}\left(\boldsymbol{b}+\frac{z}{2}\right) U^{[-]} F^{+i}\left(\boldsymbol{b}-\frac{z}{2}\right)\right]\right| P-\frac{\Delta}{2}\right\rangle,
$$

where $U^{[ \pm]}$is the future (past)-pointing $U$-shaped Wilson line along the light-cone which makes the nonlocal operator gauge invariant. At small- $x$ one may approximate $e^{-i x P^{+} z^{-}} \approx 1$ and rewrite (2.1) as

$$
x W(x, \boldsymbol{k}, \boldsymbol{b})=\frac{2 N_{c}}{\alpha_{s}} \int \frac{d^{2} \boldsymbol{r}}{(2 \pi)^{2}} e^{i \boldsymbol{k} \cdot \boldsymbol{r}}\left(\frac{1}{4} \nabla_{\boldsymbol{b}}^{2}+\boldsymbol{k}^{2}\right) S_{Y}(\boldsymbol{r}, \boldsymbol{b})
$$


where $S_{Y}(\boldsymbol{r}, \boldsymbol{b})(Y=\ln 1 / x$ is the rapidity) is the so-called dipole $\mathrm{S}$-matrix

$$
S_{Y}(\boldsymbol{r}, \boldsymbol{b})=\left\langle\frac{1}{N_{c}} \operatorname{Tr}\left[U\left(\boldsymbol{b}+\frac{\boldsymbol{r}}{2}\right) U^{\dagger}\left(\boldsymbol{b}-\frac{\boldsymbol{r}}{2}\right)\right]\right\rangle_{Y},
$$

which is the forward S-matrix of a $q \bar{q}$ pair of size $|\boldsymbol{r}|$ scattering off a hadron/nucleus at impact parameter $\boldsymbol{b}$. Note that $S$ is classically independent of $x=e^{-Y}$, but it acquires a $x$-dependence due to the quantum evolution effect. By Fourier transforming with respect to $\boldsymbol{b}$, one obtains the gluon GTMD

$$
x W(x, \boldsymbol{k}, \boldsymbol{\Delta})=\frac{2 N_{c}}{\alpha_{s}}\left(\boldsymbol{k}^{2}-\frac{\boldsymbol{\Delta}^{2}}{4}\right) \tilde{S}_{Y}(\boldsymbol{k}, \boldsymbol{\Delta}) .
$$

For an unpolarized nucleon, $\tilde{S}(\boldsymbol{k}, \boldsymbol{\Delta})$ is a function of the magnitudes $k=|\boldsymbol{k}|, \Delta=|\boldsymbol{\Delta}|$ and the relative azimuthal angle $\phi_{k \Delta}=\phi_{k}-\phi_{\Delta}$. At small-x, the dominant angular dependence is elliptic, so that we can approximately write

$$
\tilde{S}_{Y}(\boldsymbol{k}, \boldsymbol{\Delta})=\tilde{S}_{0}(k, \Delta)+2 \cos 2 \phi_{k \Delta} \tilde{S}_{1}(k, \Delta)+\cdots .
$$

Numerically, $\tilde{S}_{1}$ is at most a few percent of $\tilde{S}_{0}$ in magnitude, but has very different dependences on $k$ and $\Delta$ [7]. It can thus lead to distinct experimental signatures as we discuss shortly.

Eq. (2.4) shows that the problem of measuring the Wigner distribution reduces to that of finding a process which is sensitive to both the size $\boldsymbol{r}$ and impact parameter $\boldsymbol{b}$ of the dipole S-matrix. In order to be sensitive to $\boldsymbol{b}$, the process has to be diffractive: The proton scatters elastically with momentum transfer $\boldsymbol{\Delta}$. In order to be sensitive to $\boldsymbol{r}$, one should probe the relative momentum between the quark and antiquark. A process which meets these criteria is exclusive diffractive dijet production in $e p$ collisions [6] where the virtual photon splits into a $q \bar{q}$ pair (dipole) and scatters off the proton. The pair is then detected as a dijet in the forward region with the total transverse momentum $\boldsymbol{k}_{1}+\boldsymbol{k}_{2}=-\boldsymbol{\Delta}$ and the relative momentum $\boldsymbol{P}=\frac{1}{2}\left(\boldsymbol{k}_{2}-\boldsymbol{k}_{1}\right)$. The cross section can be written as, for the transversely polarized virtual photon,

$$
\frac{d \sigma}{d y_{1} d^{2} \boldsymbol{k}_{1} d y_{2} d^{2} \boldsymbol{k}_{2}} \propto\left|\int d^{2} \boldsymbol{q} \tilde{S}(\boldsymbol{q}, \boldsymbol{\Delta}) \frac{\boldsymbol{P}-\boldsymbol{q}}{(\boldsymbol{P}-\boldsymbol{q})^{2}+\varepsilon^{2}}\right|^{2},
$$

where $\varepsilon^{2} \propto Q^{2}$ (photon virtuality). For generic values of $Q^{2}$, it is difficult to extract $\tilde{S}$ from the measured cross section by inverting the convolution integral over $\boldsymbol{q}$. To overcome this difficulty, Ref. [6] suggested to look at the small- $Q^{2}$ region. There the $\boldsymbol{q}$-integral receives dominant contributions from $\boldsymbol{q} \sim \boldsymbol{P}$ and the cross section roughly goes as $\tilde{S}(\boldsymbol{P}, \boldsymbol{\Delta})$ squared. We then see from Eq. (2.5) that the cross section has a $\cos 2 \phi$ azimuthal angle modulation

$$
\frac{d \sigma}{d y_{1} d \boldsymbol{k}_{1} d y_{2} d \boldsymbol{k}_{2}}=d \sigma_{0}+2 \cos 2\left(\phi_{P}-\phi_{\Delta}\right) d \sigma_{1}
$$

To make the this argument more precise, let us consider the limit $Q^{2} \rightarrow 0$. Substituting (2.5) into (2.6), one can perform the angular integral analytically

$$
\int d^{2} \boldsymbol{q} \tilde{S}(\boldsymbol{q}, \boldsymbol{\Delta}) \frac{\boldsymbol{P}-\boldsymbol{q}}{(\boldsymbol{P}-\boldsymbol{q})^{2}}=-\frac{2 \pi \boldsymbol{P}}{P^{2}}\left(A+\cos 2\left(\phi_{P}-\phi_{\Delta}\right) B\right),
$$


where

$$
A(P, \Delta)=-\int_{0}^{P} d q q \tilde{S}_{0}(q, \Delta), \quad B(P, \Delta)=\int_{0}^{\infty} d q q \tilde{S}_{1}(q, \Delta)\left(-\frac{q^{2}}{P^{2}} \theta(P-q)+\frac{P^{2}}{q^{2}} \theta(q-P)\right)
$$

If one knows $A$ and $B$, one can determine $\tilde{S}_{0,1}$ by inverting the $q$-integrals in (2.9). Indeed, $\tilde{S}_{0}$ is trivially obtained as $\tilde{S}_{0}=-\frac{1}{P} \frac{\partial A}{\partial P}$. Reconstructing $\tilde{S}_{1}$ from $B$ is more tricky, but it can be done exactly [9]. The result is

$$
\tilde{S}(P, \Delta)=-\frac{\partial B(P, \Delta)}{\partial P^{2}}+\frac{2}{P^{2}} \int_{0}^{P^{2}} \frac{d P^{\prime 2}}{P^{\prime 2}} B\left(P^{\prime}, \Delta\right) .
$$

Experimentally, the region $Q^{2} \approx 0$ can be studied in the photoproduction limit of lepton-nucleon scattering. Another very interesting possibility is ultraperipheral nucleon-nucleus collisions [9] in which the nucleus is treated as a source of Weiszäcker-Williams photons which are almost real. In the latter case the cross section is given by

$$
\begin{aligned}
\frac{d \sigma^{p A}}{d y_{1} d \boldsymbol{k}_{1} d y_{2} d \boldsymbol{k}_{2}} & =\int d \omega \frac{d N}{d \omega} \frac{d \sigma^{p \gamma}}{d y_{1} d \boldsymbol{k}_{1} d y_{2} d \boldsymbol{k}_{2}} \\
& \propto \frac{d N}{d \omega}\left(A^{2}+2 A B \cos 2\left(\phi_{P}-\phi_{\Delta}\right)\right),
\end{aligned}
$$

where $\omega$ is the photon energy, and $\omega=\frac{1}{2}\left(k_{1} e^{y_{1}}+k_{2} e^{y_{2}}\right)$ in the second line. The photon flux $\frac{d N}{d \omega}$ is calculable from QED and is enhanced by the atomic number squared $Z^{2}$. This compensates the smallness of the electromagnetic coupling, making the observable particularly promising for the measurement of the gluon Wigner/GTMD distribution.

\section{Gluon orbital angular momentum at small- $x$}

Finally, we consider the case in which the nucleon is longitudinally polarized and discuss the gluon orbital angular momentum (OAM) [10]. The relation between the OAM and the Wigner distribution (1.1) actually holds at the density level

$$
L_{g}(x)=2 \int d \boldsymbol{b} d \boldsymbol{k}(\boldsymbol{b} \times \boldsymbol{k})_{z} W_{g}(x, \boldsymbol{k}, \boldsymbol{b}) .
$$

The gauge invariant 'OAM parton distribution' $L_{g}(x)$ was originally defined in [11] in a different manner, but it actually coincides with the above, more intuitive definition. The small- $x$ behavior of $L_{g}(x)$ can be studied in a similar vein to the unpolarized case discussed in the previous section, but there is an important difference. If one makes the approximation $e^{-i x P^{+} z^{-}} \approx 1$ which led to (2.2), one loses all information about the longitudinal spin. In order to obtain nonvanishing $L_{g}(x)$, one has to keep the first subleading correction $e^{-i x P^{+} z^{-}} \approx 1-i x P^{+} z^{-}$. Importantly, due to the factor $z^{-}, L_{g}(x)$ can no longer be expressed solely in terms of 'infinite' Wilson lines $U(\boldsymbol{x})=U_{\infty,-\infty}(\boldsymbol{x})$ which go from $z^{-}=-\infty$ to $z^{-}=+\infty$. Instead, it inevitably involves operators which consist of 'half-infinite' Wilson lines such as

$$
\operatorname{Tr}\left[U(\boldsymbol{x}) U_{-\infty, z^{-}}(\boldsymbol{y}) D_{i} U_{z^{-}, \infty}(\boldsymbol{y})\right]
$$


While this looks unfamiliar, remarkably, the same operator shows up in the analysis of the polarized gluon distribution $\Delta G(x)$ at small- $x$. This means that there is a linear relation between $L_{g}(x)$ and $\Delta G(x)$

$$
L_{g}(x)=-2 \Delta G(x)+\cdots .
$$

In Ref. [10], it has been argued that the neglected terms in (3.3) is small. If this is indeed the case, (3.3) has significant implications for the nucleon spin sum rule. Currently there are huge uncertainties in the gluon helicity $\Delta G=\int_{0}^{1} d x \Delta G(x)$ from the small- $x$ region. If, in future, the small- $x$ contribution to $\Delta G$ is precisely determined and turns out to be large, one should expect an even larger contribution with an opposite sign from the gluon OAM in the same $x$-region.

In conclusion, the partonic Wigner distribution is no longer a purely theoretical object. At least the gluon Wigner distribution can be measured experimentally, and a first proposal about the measurement of the quark Wigner distribution has appeared very recently. These distributions contain even richer information about the nucleon tomography than TMDs and GPDs. It is thus very interesting to explore this direction in the future experiments at the Electron-Ion Collider (EIC).

\section{References}

[1] A. V. Belitsky, X. d. Ji and F. Yuan, Phys. Rev. D 69, 074014 (2004) doi:10.1103/PhysRevD.69.074014 [hep-ph/0307383].

[2] S. Meissner, A. Metz and M. Schlegel, JHEP 0908, 056 (2009) doi:10.1088/1126-6708/2009/08/056 [arXiv:0906.5323 [hep-ph]].

[3] Y. Hagiwara and Y. Hatta, Nucl. Phys. A 940, 158 (2015) doi:10.1016/j.nuclphysa.2015.04.005 [arXiv:1412.4591 [hep-ph]].

[4] C. Lorcé and B. Pasquini, Phys. Rev. D 84, 014015 (2011) doi:10.1103/PhysRevD.84.014015 [arXiv:1106.0139 [hep-ph]].

[5] Y. Hatta, Phys. Lett. B 708, 186 (2012) doi:10.1016/j.physletb.2012.01.024 [arXiv:1111.3547 [hep-ph]].

[6] Y. Hatta, B. W. Xiao and F. Yuan, Phys. Rev. Lett. 116, no. 20, 202301 (2016) doi:10.1103/PhysRevLett.116.202301 [arXiv:1601.01585 [hep-ph]].

[7] Y. Hagiwara, Y. Hatta and T. Ueda, Phys. Rev. D 94, no. 9, 094036 (2016) doi:10.1103/PhysRevD.94.094036 [arXiv:1609.05773 [hep-ph]].

[8] D. T. Smithey, M. Beck, M. G. Raymer, and A. Faridani, Phys. Rev. Lett. 70, 1244 (1993).

[9] Y. Hagiwara, Y. Hatta, R. Pasechnik, M. Tasevsky and O. Teryaev, arXiv:1706.01765 [hep-ph].

[10] Y. Hatta, Y. Nakagawa, B. Xiao, F. Yuan and Y. Zhao, arXiv:1612.02445 [hep-ph].

[11] Y. Hatta and S. Yoshida, JHEP 1210, 080 (2012) doi:10.1007/JHEP10(2012)080 [arXiv:1207.5332 [hep-ph]].

[12] S. Bhattacharya, A. Metz and J. Zhou, arXiv:1702.04387 [hep-ph]; S. Bhattacharya, in these proceedings. 